\title{
No process for initiative
}

\author{
G J Parkes ${ }^{1}$
}

1710 Naval Air Squadron, Royal Navy

Corresponding author. Email: graham.parkes412@mod.gov.uk

\section{Synopsis}

\begin{abstract}
Throughout naval aviation engineering history, processes have continually developed and improved safety, especially with rapidly evolving complex aircraft systems. With more processes to follow, technicians will become more reliant on those processes. The post Hadden-Cave era has led to increased understanding of accountability, thereby diminishing initiative or the application of engineering principles to solve real life engineering problems. High level policy and accountability rhetoric have cascaded throughout engineering management to engineering technicians, with the warning 'do not deviate from process' from fear air safety may be compromised. This, coupled with the perceived threat of consequence to the individual should compliance be ignored, leads to a culture of process rather than a culture of lateral thought around complex engineering issues. Consequently, naval engineers feel unable to innovate or improvise practical solutions to problems despite engineers understanding the need to maintain air safety. As a war-fighting organisation which extends to disaster relief operations or damage control at sea, the supervisor/team leader may find themselves without a process and potentially unable to respond with confidence to the situation at hand. A key attribute of a Petty Officer is initiative; as reliance on process increases and reliance on effective intelligence decreases, are the Royal Navy limiting their future leaders? Attitudes and competence take time to change and develop; there is an increasing risk that the naval engineer of the future fails to think around problems and it is imperative that we encourage and develop initiative in naval aviation engineers.
\end{abstract}

Keywords: initiative; engineering innovation; process; aviation maintenance; human factors

\section{Introduction}

We live within a society where processes are fundamental in any organisation to achieve safe and effective tasks. Military aircraft maintenance is embedded within such an organisation, where maintenance procedures are the end result from structured regulation. The subsequent formation of the Military Aviation Authority (MAA) and Duty Holder construct has led to a better understanding of accountability and increased air safety that has also simplified regulation. Naval engineering technicians carry out aircraft maintenance, at home and in all environments at sea and abroad. These naval ratings undergo generic training to develop their initiative and problem solving acumen to respond to changes from the norm in order to provide practical solutions. As part of a warfighting organisation, Royal Naval air engineering sections are expected to provide serviceable aircraft. Engineering problems may need to be solved quickly in the field by responding to challenges as they arise. This paper will highlight the human factors impact, since the formation of the MAA, of reduced engineering problem solving abilities through lack of applied initiative and innovation. In particular this will focus upon the Royal Naval Air Engineering Petty Officer (PO) employed on Squadrons. The PO is an experienced supervisor and a technical subject matter expert by aircraft type and mark. And yet may not have the confidence to provide innovative solutions to engineering problems. Innovation should be considered when appropriate. Processes are fundamental to safe and effective maintenance and should be adhered to. Unfortunately, process was ignored and consequently became a causal factor to the crash of Nimrod aircraft XV230 and the death of all 14 service personnel on board that was borne out in the Nimrod Report (Hadden-Cave, 2009).

\section{The Military Aviation Authority and engineering processes}

Hadden-Cave highlighted in the Nimrod Report (Hadden-Cave, 2009) the lack of process being followed within the Nimrod Integrated Project Team, which was a reflection throughout the wider Ministry of Defence (MOD). The importance of following process was an important aspect within this report. There are four key principles of air safety in the Military Aviation Regulation: Leadership, Independence, People and Simplicity (MOD, 2014a). In particular the latter two are of interest: People. There must be much greater focus on people in the delivery of high standards of safety and airworthiness (and not just on process and paper). Simplicity. Regulatory structures, processes and rules must be as simple and straightforward as possible so that everyone can understand them. This is to say that processes are carried out free from interpretation, at a Squadron working

${ }^{1}$ Chief Petty Officer Graham Parkes is an Aircraft Engineering Technician (avionics) Royal Navy. He has over 4 years' experience facilitating human factors in various Units in the Fleet Air Arm and is currently the lead facilitator at 1710 Naval Air Squadron. He has previously been employed as a Flight Senior Maintenance Rating managing a Flight's engineering section within the Commando Helicopter Force. 
level, and are fit for purpose to achieve safe and effective aviation maintenance. People are suitably trained, experienced and authorised to carry out a role within their area of responsibility and challenge anything they consider unsafe to maintain the integrity of air safety. Continuing Airworthiness for in-service aircraft is the responsibility of the Type Airworthiness Authority including the review of maintenance procedures (MOD, 2014b) to ensure their quality. Despite the plethora of procedures and regulation of process management, excellence is aspirational and not the status quo. There can never be a procedure which suits every eventuality or environment. However, aircraft maintenance must occur safely and effectively, especially within a military fighting force. A process can also minimise the effects of Human Factors influences upon a maintenance task. The existence of procedures provides a means to improve safety, by reducing the likelihood of error, and increase productivity, especially with more complex aircraft systems.

\section{Leadership and initiative}

An aspect of Naval leadership is to project influence onto subordinates to engender within them the confidence that breeds initiative; a continuous process throughout training and everyday tasks (MOD, 2017). A Naval rating is exposed to initiative training at milestones throughout their career; Initial Naval General Training upon entering the Service, Able Rate Leadership Course during initial trade training, upon selection for promotion during the Leading Rate Leadership and the PO Leadership Courses. Leadership training includes aspects which are achievable through the application of initiative, in the form of Leadership Tasks. These tasks assess the trainee's leadership acumen, facilitated by initiative thinking. Leadership Tasks develop the individual's concept of problem solving, in a generic environment, which is a transferable skill to the rating's professional trade. At least 2 years elapse between leadership courses (MOD, 2016), although this is typically longer. Continuation Training occurs periodically to suit the professional needs for each Unit. In this author's experience application of engineering innovation Continuation Training has occurred twice in two separate Units over a 22 year career. Upon both of these occasions, with whole Unit participation, engineering principles were applied to achieve the task with varying measures of success. During routine daily employment, ratings are exposed to, influenced by and, for Leading Hands and above, practice leadership and therefore develop their leadership acumen. Within a Squadron and prior to deviation from process, the deviation must be assessed and correctly authorised. Although necessary, this has discouraged innovative style of thinking. This has led to less trial and error, application, development, influence and exposure of initiative thinking occurring within Squadrons. Therefore, the Royal Navy is potentially limiting its future leaders in applying initiative to solving engineering problems.

\section{Investigation focus group}

The focus group for this paper is the PO Aircraft Engineering Technician. The PO is usually an experienced supervisor and a technical subject matter expert by aircraft type and mark, within their engineering source trade mechanical or avionics. A PO has undertaken all rating's leadership courses and has applied leadership in the field. A trained Chief Petty Officer (CPO or Chief) Aircraft Engineering Technician however, in the role of the Senior Maintenance Rating (SMR) is authorised to make airworthiness decisions. This is necessitated by problem solving and risk mitigation. A leader should apply initiative to solve problems and development should start early in a career and not begin training as a Chief. Time is required to develop initiative thinking through trial and error. The CPO should be more of a mentor to develop these skills within subordinates. Therefore the PO must be capable of providing innovate solutions and continuously improve to allow themselves, upon promotion, to be competent and have the confidence to authorise sound airworthy decisions.

\section{Investigation and findings}

The purpose of this investigation is to discuss; the views of Squadron maintenance POs on the interaction between engineering processes and initiative, their views upon the effectiveness of processes and the application of initiative to overcome shortfalls or improvements upon those processes, their views on the encouragement received from senior engineering management to problem solve, and throughout this paper to identify any trends.

To achieve this, findings have been compiled from two sources: human factors facilitated sessions and by questionnaire. The author has facilitated Human Factors session within the Military Air Environment (MAE), predominately in the Squadron environment. Training recipients from the wider MAE are air engineering personnel who have also worked within the Squadron environment. The Human Factors facilitations were discussion based with a prior agenda. Although in the majority of these facilitations, discussing initiative and process-driven maintenance became a consequence of the points discussed.

In a recent survey sent out to air engineering POs over two Squadrons, 27 responses were returned. The qualitative questionnaire contained nominal labels and up to a 5-point Likert Scale of ordinal data points including the opportunity to provide additional information. 
Both sources of data discussed process and initiative. The following findings are structured to the questionnaire format to provide a logical flow of findings from both sources.

\subsection{Need for processes}

Both sources agreed that processes are necessary and are generally fit for their intended purpose. During the sessions it was apparent, due to new aircraft entering service, that processes were essential because of the complexity of modern and integrated systems. For example, navigating through multi-functional displays to find the necessary maintenance page or system test required a specific set up that was not intuitive. For many, procedures for routine maintenance activities were used as a checklist for repetitive work. There was some frustration due to several processes that needed to be followed. Although there were no reports of contradiction, there were several procedures to follow to achieve similar tasks. During a session offered by a supervisor, a junior maintenance rating had followed the process explicitly. A pipeline that had become loose, located in the immediate area of the mandatory checks being carried out, was not identified as the procedure did not call for it to be checked. Although the junior maintainer had carried out this task correctly, many supervisors felt an amendment to the procedure would prevent future mishaps, leading to more procedures. There may be more similar examples of this, where a culture of process is being ingrained early in a rating's career.

\subsection{Process inaccuracies}

The survey indicated a mode average of up to $10 \%$ of processes used were inaccurate, no matter how minor the inaccuracy. The overwhelming contributory cause to these oversights was the lack of equipment on the Unit to conduct the necessary task. During facilitations it became apparent that equipment was not available due to equipment unserviceability, servicing or calibration off-unit and in the majority of responses equipment had not been procured or was no longer available. With regards to the two latter reasons the process was impractical to follow. Although a mechanism to request for change (MOD, 2014b) exists there is no confidence in its use. It is felt that requested changes are often rejected, for non-safety related concerns and those requests that are accepted take an inordinate amount of time to take effect. Maintainers should have confidence in these processes, those processes must be continually developed to meet the demands of ever changing requirements in equipment, regulation and quality. Squadron maintainers are often busy in order to meet the Operational flying programme and are reliant upon process.

\subsection{Opportunities to apply initiative}

Opportunities which exist within the workplace to apply and develop initiative were viewed mostly positively. Facilitated sessions provided several examples where innovation could improve everyday activities within the workplace. Examples included: improving the movement of aircraft in and out of the hangar; and using ground servicing equipment, such as a power rig, instead of the recommended ladder when unavailable. In every example the engineers could justify reasoning behind their statements. The survey split this question into two parts. The first part asked whether generic leadership opportunities to apply initiative exist within the workplace. The second part questioned whether the respondent could apply initiative to overcome maintenance problems. The sessions were mainly corroborated by the survey, where a modal average agreed. There was however, a potentially concerning trend from the respondents who joined the Royal Navy between 2006 and 2009. These responses ranged predominately between strongly disagree, disagree and neutral. Although the responses to this nominal label were few, there exists a potential lack of initiative thinking of air engineers joining the Royal Navy post the formation of the MAA in 2010, this is expressed in Figure 1. This may support reliance being placed in increasing process and as a consequence reduces opportunities to offer effective intelligence to solve problems that are becoming the attitudinal norm. 
Joined RN prior to 2005

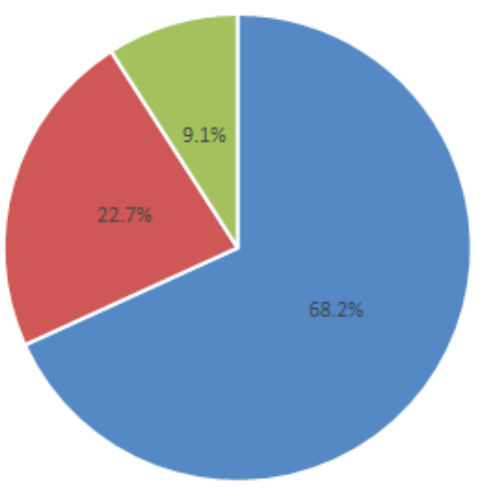

Joined RN between 2006-2009

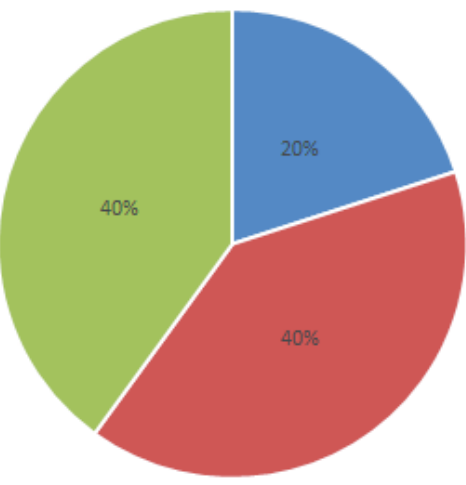

- Strongly agree or agree | Neutral || strongly disagree or disagr ee

Figure 1: Survey responses to the question: 'Many opportunities exist in your workplace to apply initiative' demonstrating the differing nominal labels: joining prior to 2005 and joining between 2006 and 2009.

\subsection{Initiative confidence}

The confidence to apply initiative was mixed during the facilitated sessions. The Chiefs and senior POs within group discussions, were able to provide examples of a time that they had applied engineering initiative within the workplace to overcome certain issues. The majority of examples given were of the mis-application of equipment and tooling to provide a purpose other than that it was intended for, due to no suitable equipment being available. For example, applying wire to lock the tangs of multiple P-clips to line-up the holes because standard techniques proved ineffective for this particular task. A Royal Navy Reservist offered that whilst on Operational service he had been authorised to investigate a fault and repair the electronic circuit, without circuit diagrams, within a system component whilst at sea; this allowed the application of engineering principles in a realistic environment. The relatively junior POs offered that any type of issue relating to process, lack of equipment or other technical issue is problem solved by the Squadron's engineering management team or through the Technical Query Service. The technical query would allow the Force Technical Support Team to manage the issue. It was also stated that problem solving is taken away from Squadron engineers and given to outside agencies.

The contributory factor for this deviation between the two groups of seniority is due to attitudes of employment significantly before and after the formation of the MAA. A Chief offered a reason to the deviation: the senior group joined the Royal Navy during the 1990s and in some cases the 1980s. Although process did exist, the need to achieve the task was the driving force. Initiative was expected and encouraged and a necessity for a Senior Rate that was seen as an attitudinal norm.

In the post Hadden-Cave era, the attitudinal norm is to follow process and apply less problem solving within the Squadron which this data shows to be done by the involvement of outside agencies. The Royal Navy is a warfighting organisation and throughout a period of Operational service, especially at sea, solving problems may need to happen quickly. There may not be sufficient time for effective problem solving from external agencies. Further to this, the ship may be tactically radio silent and therefore no communication is possible with outside agencies. Squadron personnel must therefore provide effective solutions to problems. However, in this scenario the PO may find themselves without a process and perhaps unable to respond with confidence to apply initiative as there has been no previous experience.

A veteran of the Falklands Campaign provided examples of pragmatic innovative engineering solutions. The Automatic Flying Control System Amplifier internal power supply unit, installed in the Sea King Helicopter, converted 115VAC to 12VDC during normal use. This power supply unit had failed with no authorised spare parts available. It was vital that the Anti-Submarine Warfare capable aircraft was made ready for Operational tasking. The engineering team considered an electric shaver's power supply as a potential alternative part. A bench test confirmed the shaver's capability to perform to the amplifier's specification and subsequently became a viable solution without the need of external advice.

\subsection{Provide solutions through the Chain of Command and initiative competence}

The questionnaire asked 'How often do you offer/provide solutions, through the chain of command, to maintenance problems?' Figure 2 provides a breakdown of the survey responses. The mode average was significantly sometimes, with far fewer never and often responses. The option for always was not ticked. The 
questionnaire also asked 'How confident do you feel should you need to apply engineering initiative in your workplace?' The response was overwhelmingly confident and very confident. There were far fewer responses for neither confident nor not confident and not confident and no returns for not very confident. This survey question did not fully support the facilitated sessions. From the survey there were no responders who had joined the Royal Navy from 2010 onwards. The questionnaire nominal labels provided options of joining the Royal Navy 2005 or prior and 2006-2009, therefore those that had joined close to 2005 may have been influenced by current attitudes. The facilitated sessions provided diversity in seniority and experience that the questionnaire did not provide. Furthermore, initiative tests were not performed during sessions nor the questionnaire to determine proficiency in innovative thinking.

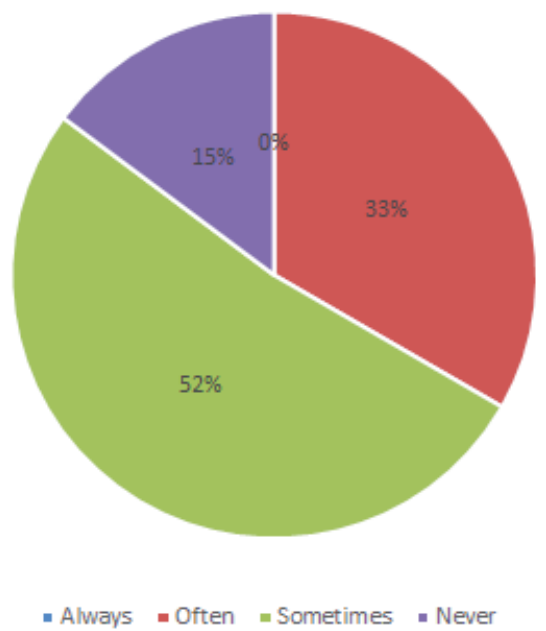

Figure 2: Survey response to the question 'How often do you offer/provide solutions, through the chain of command, to maintenance problems?'

Self-evaluation is complicated and confidence without proven competence is perceived (Stewart et al, 2003) which may have led to the high rate of a confident response despite little evidence. Confidence in using initiative is the perception of the individual and this paper provides inconclusive evidence. Innovation is sometimes offered, through the chain of command, which infers more potential innovative ideas exist. However, sessions do not fully support this especially for the less experienced POs. Therefore, innovative competence has not been proven. Innovative thinking cannot be taught, it can be developed. Competence takes time to develop and this is achievable through experience of trial and error. Without competence being proven and subsequent confidence to apply initiative, there is a risk that the future naval engineering ratings will fail to be able to think around engineering problems.

\subsection{Encouragement to offer initiative}

Facilitated sessions explored the reasons why more innovative ideas had not been raised through the Chain of Command. It became apparent that there were parallels with lack of encouragement from line management to innovation. There were varied responses from POs during the facilitated sessions with many others also agreeing with their colleagues. The foremost reason was due to busy work schedules which did not allow time to provide solutions and ideas through the Chain of Command. Other reasons offered by a Chief who was authorised to make airworthy decisions as the SMR provided an example: A PO had refused to investigate a suitable alternative grease to satisfy a maintenance task, as the specified grease was unavailable. The Chief stated that the PO felt it was not his job and this investigation should solely be carried out by those that hold the appropriate authority, although at no time was the PO expected to make an airworthy decision. Similarly some survey responses also supported that innovation was not their job. In some instances the engineering management did not wish any proposed deviation from a process that was offered by Squadron personnel, irrespective of any potential benefits that may have arisen. Any queries were forwarded on to the force technical support team for problem solving to be undertaken outside of the Squadron.

POs from one particular Unit felt undermined by their engineering management. POs are often senior and experienced supervisors and competent to conduct effective fault diagnosis. However, the subsequent work recording needed to demonstrate all work had been carried out in accordance with a referenced procedure and any absence was returned for amendment. This had led to some work recording remaining absent despite a fault being fixed as a suitable reference was not available. There were responses from Squadron-wide engineering personnel who received briefings that deviation from process must not occur, under any circumstance. Any deviation would result in disciplinary action. Although this is correct to safe-guard all concerned by limiting authorised people to 
make airworthy decisions, it was interpreted by many within that Unit that any potential thought of improvement or suggestion of innovation was exclusively discouraged for maintenance personnel. Processes which help to provide a safe system of work must not be deviated from without the appropriate level of authority in order to maintain the integrity of airworthiness. Facilitated sessions however, did provide some positive responses where initiative was encouraged by engineering management.

The survey corroborated the facilitated reasoning why maintenance personnel could not offer initiative to maintenance problems. The survey responses however, were mostly positive indicating line management encouraged the offering of solutions to maintenance problems. This may be due to routine changes of key maintenance engineering managers.

\section{Conclusion and further research}

In conclusion, it is clear processes are fundamentally fit for purpose by improving productivity and reducing the likelihood of error and thus increasing safety. There is supporting evidence that since the formation of the MAA, more problem solving is taken away from Squadron maintainers. This has had a discouraging effect upon maintainers to raise solutions through the Chain of Command, which may also be dependent upon workloads and personalities within the Unit engineering management. The latter does not imply lack of confidence in maintainers, but adherence to a structure which prevents ratings development in applying initiative. External organisations have a lack of time to problem solve as well as being uncontactable in an Operational environment. There is potential concern that ratings joining the Service post 2010 do not feel they can use initiative through indoctrination borne out from the MAA regulation - it is recommended that this becomes a quantitative study to better understand the current ability to initiative application. There is, therefore, a risk that future naval ratings feel unable to offer initiative to practical solutions. If nothing will change, external problem solving will become the attitudinal norm without further thought of a solution. POs should have the confidence achieved through competence to provide innovation solutions and be ready to fight and win during a crisis period.

\section{References}

Hadden-Cave, C. (2009). An independent review into the broader issues surrounding the loss of the RAF Nimrod MR2 Aircraft XV230 in Afghanistan in 2006, London: The Stationary Office, pp. 9, 163, 317, 321.

MOD, 2014,

a. Military Aviation Authority 01: Military Authority Regulatory Policy issue 6, p.8.

b. Manual of maintenance and airworthiness process-01 (MAP-01) issue 7.1, ch 5.1, p.5 and ch 8.2

MOD, 2016, BR3(1) Naval Personnel Management version 7, article 7607 [restricted access].

MOD, 2017, Joint Doctrine Publication 0-10: Maritime Power (fifth edition). p.33.

Stewart, J., O’Halloran, C., Barton, R., Singleton, S., Harrigan, P., Spencer, J., (2003). Clarifying the concepts of confidence and competence to produce appropriate self-evaluation measurement scales.

\section{Bibliography}

Bennett, A., Hellier, E. and Weyman, A., (2015). Forthcoming. Characterising influences on safety culture in military aviation: a methodically grounded approach.

Chell, E., Athayde, R., (2009). The identification and measurement of innovative characterises of young people.

East Carolina University, (2015). What is a Likert Scale. [online] Available at: http://core.ecu.edu/psyc/wuenschk/StatHelp/Likert.htm [Accessed 9 Apr. 2018]

Process Excellence Network, (2012). A brief history of process: from the industrial revolution to today. [online] Available at: https://www.processexcellencenetwork.com/lean-six-sigma-business-transformation/articles/abrief-history-of-process-from-the-industrial-re [Accessed 9 Apr. 2018]

Shuspace, Questionnaire design and analysing the data using SPSS. [online] Available at: https://students.shu.ac.uk/lits/it/documents/pdf/questionnaire_analysis_using_spss.pdf [Assessed 3 Apr. 2018] 
SurveyKing, (2018). Likert Scale explanation \& interactive example. [online] Available at: https://wwww.surveyking.com/help/likert-scale-example [Accessed 10 Apr. 2018] 